\title{
Influence of the Different Prosthesis Designs on Subjective Functional Evaluation after TKA: Medium-Term Outcome Using the 2011 Knee Society Score
}

\author{
Hiroaki Kanazawa*, Yuichiro Maruyama \\ Department of Orthopaedic Surgery, Juntendo University Urayasu Hospital, Chiba, Japan \\ Email: `hiroakimed@yahoo.co.jp
}

How to cite this paper: Kanazawa, H. and Maruyama, Y. (2020) Influence of the Different Prosthesis Designs on Subjective Functional Evaluation after TKA: Medium-Term Outcome Using the 2011 Knee Society Score. Open Journal of Orthopedics, 10, 371-383. https://doi.org/10.4236/ojo.2020.1012035

Received: November 1, 2020

Accepted: December 13, 2020

Published: December 16, 2020

Copyright (อ 2020 by author(s) and Scientific Research Publishing Inc. This work is licensed under the Creative Commons Attribution International License (CC BY 4.0).

http://creativecommons.org/licenses/by/4.0/ (c) (i) Open Access

\begin{abstract}
Background: Patient-reported outcome (PRO) has become the focus of increased attention in the assessment of total knee arthroplasty (TKA). The purpose of this study was to investigate whether different prosthesis designs affected postoperative patient-reported performance. Materials and Methods: We reviewed 216 patients (234 knees) implanted with the Press-Fit Condylar $<$ PFC $>$ Sigma prosthesis at our institution between January 2009 and December 2011. This study included 76 knees with fixed-bearing cruciate-retaining (FB-CR), 78 knees with fixed-bearing posterior-stabilized (FB-PS), and 80 knees with mobile-bearing posterior-stabilized (MB-PS) designs. The mean follow-up was $8.0 \pm 0.74$ years. Preoperative and follow-up ratings according to the 2011 Knee Society Score (2011 KSS), range of motion (ROM), and standing femoro-tibial angle (FTA) were obtained for all patients. Additionally, Western Ontario and McMaster Universities Arthritis Index (WOMAC) scores were collected at last follow-up. Results: Three separate subscales of the $2011 \mathrm{KSS}$ of the three cohorts were significantly improved post-operatively compared to the pre-operative values $(p<0.05)$, except for expectation scores. At the time of follow-up, significantly higher the 2011 Knee Society Functional Scores $<2011$ KSFS $>(P=0.016)$ were found in the PS group. In particular, there were significant differences in advanced activities of the 2011 KSFS among the three groups $(P=0.017)$. Satisfaction scores showed no difference among the three groups $(P=0.251)$. On the other hand, WOMAC and expectation scores were significantly better in the PS groups. $(P=0.003, P=0.004)$. ROM in the MB-PS group $\left(124.0^{\circ}\right)$ was better than that in FB-PS $\left(119.4^{\circ}\right)$ and FB-CR $\left(118.9^{\circ}\right)(P=0.005)$. On the other hand, additional surgery was needed in five PS knees. Conclusion: The PS
\end{abstract}


prostheses had superior $2011 \mathrm{KSFS}$, expectation scores, WOMAC scores and ROM than the CR prostheses. In contrast, the postoperative prosthesis-related complication rates were lower with $\mathrm{CR}$ prostheses. Further detailed evaluation is necessary to determine whether the characteristics of the different prostheses affect PRO.

\section{Keywords}

Total Knee Arthroplasty, Patient-Reported Outcome, 2011 New KSS, Prosthesis Design

\section{Introduction}

Total knee arthroplasty (TKA) is considered a very successful treatment for the reduction of pain and physical impairment in patients with end stage osteoarthritis $(\mathrm{OA})$. However, a proportion of patients complain of persistent or recurrent pain and dissatisfaction after TKA. Furthermore, patients increasingly expect to be more active and pain-free after surgery. In particular, Asian patients require deep flexion due to their daily lifestyles. Improvements of functional ability are often considered the most important factors after TKA, and patients' own assessment of these outcomes is a key element in evaluating the effectiveness of the procedure. Therefore, we consider that it is important to evaluate functional performance after TKA in more detail. Furthermore, it remains unclear whether the choices of prosthesis design influence patient-reported performance after TKA.

If retaining the posterior cruciate ligament (PCL) enhances joint sensation, this may explain the slightly improved functional scores for gait and stair-climbing associated with cruciate-retaining (CR) designs. Mobile-bearing (MB) prostheses offer greater conformity and decreased contact stresses through a polyethylene liner that is mobile relative to the tibial tray. This increased contact area potentially results in decreased polyethylene wear. Most of MB prostheses have been developed with the objective of realizing these theoretical advantages, but it is unclear whether these advantages translate into better clinical outcome. In contrast, the potential complication of this design includes patellar clunk and bearing dislocation [1].

In previous study, many articles regarding comparative clinical studies of Press Fit Condylar $<$ PFC $>$ Sigma prostheses design reported no significant difference in evaluation using the conventional Knee Society Score (Insall et al. 1989) [2]. However, the 1989 Knee Society Score (The 1989 KSS) are only physician-reported scores. The new Knee Society Score, copyrighted in 2011, is a validated system that combines a physician-derived component with a patient-derived component. [3] Therefore, the 2011 Knee Society Score (The 2011 KSS) can evaluate the postoperative condition much more specifically than the 1989 KSS. Moreover, it is important to consider medium-term outcome of dif- 
ferent prosthesis designs, as implant design may confer an advantage in terms of functional performance.

To our knowledge, there is little literature on medium-term patient-reported outcomes (PRO) using the 2011 KSS regarding different type of PFC Sigma prostheses. Therefore, the purpose of this retrospective study was to compare medium-term PRO among the three types of PFC-Sigma prosthesis. This study was approved by our local research ethics committee.

\section{Materials and Methods}

We reviewed 271 consecutive knees in 259 Japanese TKA patients at our institution between January 2009 and December 2011. The inclusion criteria were a diagnosis of OA or osteonecrosis with an indication of TKA. The prostheses used in this study were PFC Sigma series (Depuy-Synthes Inc. Warsaw, Indiana, USA). Both the FB-CR and the FB-posterior-stabilized (PS) of the same components possessed basically the same surface geometry, except that a post-cam mechanism was added to the PS type. In addition, we used a MB-PS, rotating-platform flex (RP-F) PFC Sigma.

There were no specific criteria for prosthesis selection, as the surgeon did not selectively use the prosthesis design based on specific preoperative conditions. The three groups represent three consecutive cohorts of patients, as the primary surgeon gradually changed his practice and preference. The use of one or other prosthesis type was chosen by alternating their deployment in patients and not for clinical reasons; in the first, fourth and seventh cases of each day, one type of prosthesis was used, while the other type was used in the second, fifth, eighth cases, and 3,6, 9 cases. The exclusion criteria were $\geq 30^{\circ}$ flexion contracture, $\geq 20^{\circ}$ varus deformity, $\geq 10^{\circ}$ valgus deformity, and significant bone defects of the femur and/or tibia requiring component augmentation. Furthermore, two patients with hemiparalysis due to brain infarction and four patients with OA of the hip or ankle were excluded, and there were 29 (10.7\%; 29/271) incomplete discretionary activities in the 2011 KSS, two cases of which required augmentation. In total, 37 knees were excluded. Finally, 234 knees in 216 patients met the inclusion criteria (76 knees with FB-CR, 78 knees with FB-PS, and 80 knees with MB-PS) (Figure 1).

\subsection{Surgical Procedure}

Operative anesthesia was either general or spinal. All the procedures were performed by two surgeons. In all knees, an anterior midline skin incision $(10 \mathrm{~cm}$ in length) was used under a pneumatic tourniquet, followed by a mid-vastus approach. Femoral preparation was performed initially, followed by tibial preparation. First, the osteophytes were removed, and the distal femoral resection was set at 5 - 7 degrees of valgus. We attempted to set $3^{\circ}-5^{\circ}$ external rotation of the femoral component in relation to the posterior aspect of the femoral condyles using preoperative computer tomography $(\mathrm{CT})$. We used the modified-gap 


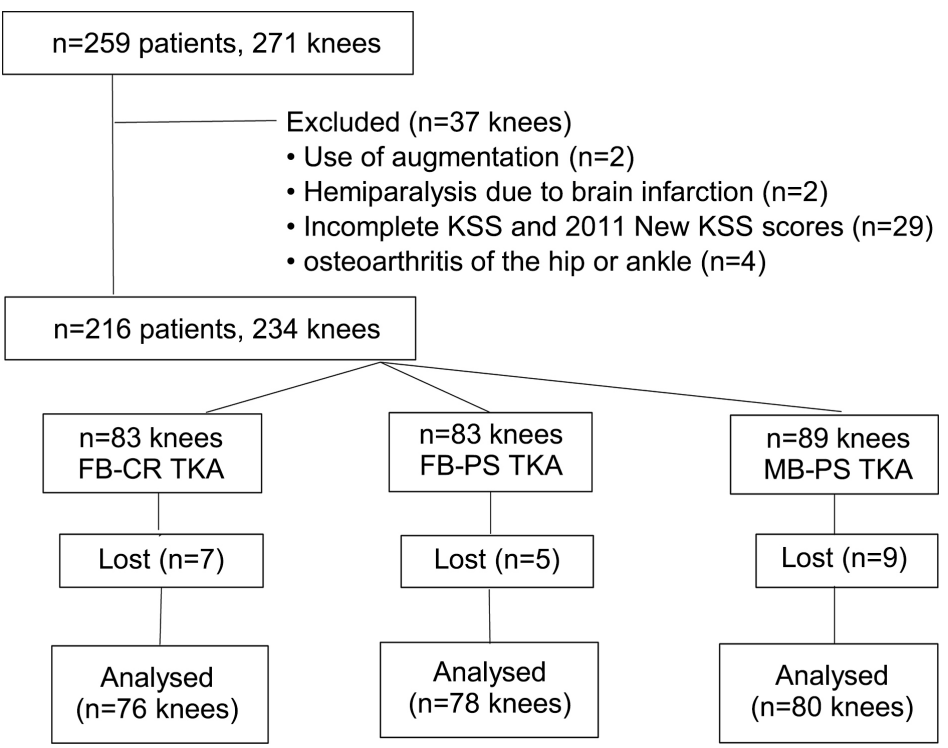

Figure 1. Participant flow chart for the study.

technique intraoperatively. The tibia was cut perpendicular to its axis, and ligament balancing was performed to achieve a balanced flexion and extension gap and restoration of the anatomical axis of the limb. All the components were cemented, and all patellae were routinely resurfaced with use of a domed, all-polyethylene patellar component. One deep drain was left in the knee for 24 hours.

After surgery, a continuous passive-motion machine was utilized. On the second postoperative day, all patients began either standing at the bedside or walking with use of crutches or a walker, as well as ROM exercises. Patients were randomized to receive edoxaban tosylate hydrate $30 \mathrm{mg}$ once daily for 10 days following TKA as prophylaxis for deep vein thrombosis (DVT).

\subsection{Evaluation}

Post-operatively, patients were evaluated at 3,6 , and 12 months, and annually thereafter. All patients were assessed clinically and radiologically prior to surgery, and at a mean follow-up time of $8.0 \pm 0.74$ years after surgery. In the present study, we assessed the preoperative and follow-up evaluations according to active ROM, the 2011 KSS. Additionally, the Western Ontario and McMaster Universities Osteoarthritis Index (WOMAC) scores were compared among the three groups, collected at eight years [4]. With regard to knee motion, knee angle in a supine position was measured using a goniometer before the operation and at the time of review.

The 2011 KSS consists of 34 items and assesses four separate subscales: the objective physician reported knee score (Knee Society Knee Score: KSKS), satisfaction and expectations, and functional activities (Knee Society Functional Score: KSFS). The subjective patient-reported score has three items for symptoms, five items for satisfaction, three items for expectation and 19 items for 
functional activities, including five items for walking and standing, six items for standard activities, five items for advanced activities and three items for discretionary activities. Regarding the three items for symptoms, visual analog scale (VAS) scores are evaluated for pain when walking on level ground and on stairs or inclines, and feeling around the knee.

Regarding radiographic analysis, anterior-posterior (AP), lateral and Merchant's views, and a full-length standing radiograph were obtained preoperatively and at follow-up. These were analyzed using the KS radiological scoring system to delineate radiolucent line around the component-bone interfaces [5]. Definitive loosening was defined as a complete radiolucent line wider than $1 \mathrm{~mm}$ in all zones, and progressive radiolucent lines wider than $2 \mathrm{~mm}$. The mechanical femoro-tibial angle (mFTA) was defined as the lateral angle of the mechanical axes of the femur and tibia, and was measured on weight-bearing radiographs.

\subsection{Statistical Analysis}

ANOVA with Bonferroni's correction test was used to assess differences between preoperative and postoperative values of all continuous outcome, including the variables for $2011 \mathrm{KSS}, \mathrm{ROM}$, and mFTA. GraphPad Instat statistical software (GraphPad Software, San Diego, CA) was used to perform all analyses. $P$ values less than 0.05 were considered significant.

\section{Results}

\subsection{Preoperative and Follow-Up Evaluation}

In total, 234 knees in 216 patients ( 31 men and 185 women) were enrolled in the study. The mean age at the time of surgery was $75.2 \pm 7.0$ years (range: $68-89$ years). Body mass index (BMI) was $26.6 \pm 4.97 \mathrm{~kg} / \mathrm{m}^{2}$ (range: 18 - 37). Median body height and weight were $151 \mathrm{~cm}$ (range: 138 - 161) and $61 \mathrm{~kg}$ (range: 36 83), respectively (Table 1 ). The preoperative knee diagnoses were 218 OA (93.2\%), and 16 osteonecrosis $(6.8 \%)$. The mean postoperative follow-up period was $8.0 \pm$ 0.74 years (range: 87 - 105 months).

There were no significant pre-operative differences in demographics data of the three cohorts (Table 2).

Table 1. Pre-operative demographics data.

\begin{tabular}{ccccc}
\hline & FB-CR & FB-PS & MB-PS & \multirow{2}{*}{ P value } \\
\cline { 2 - 4 } & $\mathrm{n}=76$ & $\mathrm{n}=78$ & $\mathrm{n}=80$ & \\
\hline Age (years) & $75.8 \pm 5.5$ & $74.5 \pm 7.2$ & $75.2 \pm 8.4$ & n.s. \\
Gender (female/male) & $61 / 15$ & $72 / 6$ & $70 / 10$ & \\
Body mass index (BMI: kg/m) & $26.3 \pm 4.5$ & $27.6 \pm 6.0$ & $26.0 \pm 4.0$ & n.s. \\
Follow up (years) & & $8.0 \pm 0.74$ & & \\
\hline
\end{tabular}


Table 2. Pre-operative and Post-operative ROM, FTA, and the 2011 KSS.

\begin{tabular}{|c|c|c|c|c|}
\hline Pre-OP & FB-CR & FB-PS & MB-PS & $P$ value \\
\hline ROM & $119.5 \pm 9.4$ & $116.3 \pm 11.6$ & $119.4 \pm 16.0$ & n.s. \\
\hline FTA & $186.5 \pm 5.08$ & $187.5 \pm 6.02$ & $187.3 \pm 6.50$ & n.s. \\
\hline \multicolumn{5}{|l|}{$2011 \mathrm{KSS}$} \\
\hline Knee Score & $29.5 \pm 8.90$ & $24.8 \pm 17.3$ & $33.3 \pm 16.1$ & n.s. \\
\hline Symptom/25 & $9.25 \pm 3.3$ & $7.44 \pm 6.4$ & $8.12 \pm 4.1$ & n.s. \\
\hline Patient Satisfaction/40 & $15.7 \pm 6.5$ & $13.3 \pm 5.7$ & $14.0 \pm 5.7$ & n.s. \\
\hline Patient Expectaion/15 & $13.8 \pm 1.4$ & $14.5 \pm 0.5$ & $15.0 \pm 0.0$ & n.s. \\
\hline Functional score/100 & $42.6 \pm 13.7$ & $42.9 \pm 9.3$ & $40.9 \pm 13.4$ & n.s. \\
\hline Post-OP & FB-CR & FB-PS & MB-PS & $P$ value \\
\hline ROM & $118.9 \pm 10.8$ & $119.4 \pm 9.8$ & $124.0 \pm 7.5$ & $0.005^{*}$ \\
\hline FTA & $175.1 \pm 1.70$ & $175.4 \pm 2.80$ & $175.4 \pm 0.97$ & n.s \\
\hline \multicolumn{5}{|l|}{$2011 \mathrm{KSS}$} \\
\hline Knee Score & $87.2 \pm 10.6$ & $91.1 \pm 10.6$ & $90.8 \pm 5.61$ & n.s. \\
\hline Symptom/25 & $20.5 \pm 3.7$ & $20.6 \pm 4.6$ & $21.7 \pm 2.6$ & n.s. \\
\hline Patient Satisfaction/40 & $26.0 \pm 7.7$ & $29.0 \pm 6.6$ & $27.3 \pm 7.6$ & n.s. \\
\hline Patient Expectation/15 & $9.5 \pm 2.1$ & $11.1 \pm 2.0$ & $11.1 \pm 2.3$ & $0.004^{\star}$ \\
\hline Functional score/100 & $65.9 \pm 13.7$ & $73.5 \pm 12.6$ & $73.5 \pm 14.7$ & $0.016^{*}$ \\
\hline
\end{tabular}

At the time of follow-up, there was a significant difference in ROM among the groups $(P=0.005)$. ROM in MB-PS groups showed better than that in FB-PS and FB-CR groups $\left(124.0^{\circ}, 119.4^{\circ}\right.$, and $118.9^{\circ}$, respectively; Table 2$)$. Additionally, the mean 2011 KSKS were similar among the three groups. In contrast, the mean 2011 KSFS were significantly higher in the FB-PS $(73.5 \pm 12.6)$ and MB-PS $(73.5 \pm 14.7)$ groups than in the FB-CR group $(65.9 \pm 13.7)$ at the time of the last follow-up ( $P=0.016$; Table 2). In particular, the items of advanced activities in the 2011 KSFS of the MB-PS and FB-PS groups were significantly higher than those of the FB-CR group ( $P=0.017$; Table 3$)$.

The satisfaction score showed significant improvement $(P<0.001$ each) after surgery, with no significant difference among the groups (Table 2). On the contrary, expectation scores were lower at the last follow-up compared to preoperatively, and were better in the FB-PS and MB-PS groups than in the FB-CR group. $(P=0.004$; Table 2).

The scores in each category of the postoperative WOMAC are shown in Table 3. At the eight-year follow-up, the PS groups had a significant superior score in the WOMAC compared with the CR groups $(P=0.003)$. 
Table 3. Details of postoperative the $2011 \mathrm{KSFS}$ and WOMAC.

\begin{tabular}{ccccc}
\hline 2011 KSFS & FB-CR & FB-PS & MB-PS & P value \\
\hline waking and standing/30 & $20.9 \pm 7.30$ & $21.8 \pm 6.40$ & $22.5 \pm 6.30$ & n.s. \\
standard activities/30 & $23.6 \pm 3.42$ & $24.4 \pm 2.90$ & $23.8 \pm 4.30$ & n.s. \\
advanced activities/25 & $12.3 \pm 5.40$ & $15.0 \pm 4.50$ & $15.2 \pm 4.50$ & $\mathbf{0 . 0 1 7 ^ { * }}$ \\
discretional activities/15 & $11.8 \pm 2.28$ & $12.1 \pm 2.38$ & $12.0 \pm 2.82$ & n.s. \\
\hline WOMAC & FB-CR & FB-PS & MB-PS & P value \\
\hline Pain/20 & $1.63 \pm 1.77$ & $1.28 \pm 1.84$ & $0.90 \pm 0.74$ & n.s. \\
Stiff/8 & $1.88 \pm 2.64$ & $0.83 \pm 0.92$ & $0.90 \pm 0.74$ & $0.0003^{*}$ \\
Function/68 & $10.1 \pm 8.69$ & $5.72 \pm 4.97$ & $6.11 \pm 4.33$ & $0.0003^{*}$ \\
Total/96 & $12.2 \pm 4.38$ & $8.94 \pm 7.68$ & $7.18 \pm 5.44$ & $\mathbf{0 . 0 0 3 ^ { * }}$ \\
\hline
\end{tabular}

Correlation between the satisfaction, expectation score and the individual subscales of the 2011 KSFS are shown in Table 4. In this study, advanced activity score was not correlated with patient satisfaction regardless of the three different prostheses.

\subsection{Radiographic Evaluation}

Postoperative mFTA was similar among the groups (Table 2). A radiolucent line at the interface between the tibial prosthesis and the tibial bone was observed in two patients in the FB-CR group and one patient in the FB-PS group, and at the interface between the femoral prosthesis and the femoral bone in one patient in the FB-CR group and one patient in the FB-PS group. In all groups, the locations of the radiolucent lines were in zones 1,3 , and 4 , and all were incomplete, less than $2 \mathrm{~mm}$ in width, and non-progressive. No knee had loosening of the femoral, tibial, or patellar component, and patellar subluxation was observed in one patient with the FB-PS design.

\subsection{Complications}

There were no reported cases of intra-operative fracture, hematoma, or infection. Four cases (4/234: 1.7\%) had symptomatic distal DVT, but there were no cases of DVT spreading to the proximal veins and/or onset of pulmonary embolism. Also, two cases (2/234: 0.9\%) had cardiac ischemic attack. In 4/234 cases (1.7\%), partial re-suture was needed in the wounded area because of a delay in union or dehiscence. Three MB-PS knees (3/80: 3.8\%) and two FB-PS knees (2/78: $2.6 \%)$ had patellar clunk, and resolution with arthroscopic debridement was required in three knees. We experienced bearing spin-out of two MB-PS knees (2/80: $2.5 \%)$, which could not be reduced by closed manipulation, so we performed open reduction. 
Table 4. Correlation (Person product-moment) between the satisfaction, expectation score and the individual subscales of the $2011 \mathrm{KSS}$ functional activities.

\begin{tabular}{ccc}
\hline Correlation & \multicolumn{2}{c}{ r value (P-value) } \\
\hline \multicolumn{1}{c}{ Subscale } & \multicolumn{2}{c}{$2011 \mathrm{KSS}$} \\
\hline Post-OP & Satisfaction score & Expectation score \\
\hline - FB-CR groups & & \\
Functional activities & $0.6039(\mathbf{0 . 0 0 0 3})$ & $0.2655(0.1489)$ \\
waking and standing/30 & $0.5288(\mathbf{0 . 0 0 2 2})$ & $0.5743(\mathbf{0 . 0 0 0 5 )}$ \\
standard activities/30 & $0.1378(0.4599)$ & $0.6033(\mathbf{0 . 0 0 0 2})$ \\
advanced activities/25 & $0.2073(0.2631)$ & $0.3328(0.0723)$ \\
\hline discretional activities/15 & & \\
\hline
\end{tabular}

\section{- FB-PS groups}

$\begin{array}{lll}\text { Functional activities } & & \\ \text { waking and standing/30 } & 0.3007(\mathbf{0 . 0 4 4 8}) & 0.1923(0.2056) \\ \text { standard activities/30 } & 0.1730(0.2556) & 0.3773(\mathbf{0 . 0 1 0 6}) \\ \text { advanced activities/25 } & 0.0013(0.9934) & 0.0936(0.5408) \\ \text { discretional activities/15 } & 0.3102(\mathbf{0 . 0 3 8 1}) & 0.1040(0.4965)\end{array}$

- MB-PS groups

Functional activities

waking and standing/30

$0.3675(\mathbf{0 . 0 1 6 7 )}$

$0.3573(\mathbf{0 . 0 2 0 2 )}$

$0.0219(0.8905)$

$0.4232(\mathbf{0 . 0 0 5 2 )}$
$0.0661(0.6775)$

$0.2502(0.1100)$

$0.1908(0.2261)$

$0.4044(\mathbf{0 . 0 0 7 9})$

\section{Discussion}

The most important finding of this study was that the 2011 KSFS were significantly higher in the PFC Sigma PS (FB-PS, MB-PS) group than in the PFC Sigma FB-CR group. In particular, advanced activities in the 2011 KSFS showed differences among the three groups. However, we failed to prove that advanced activity score correlates with patient satisfaction regardless of the three different prostheses. Additionally, postoperative expectation scores were significantly higher in the FB- and MB-PS groups than in the FB-CR group.

In previous study, many articles regarding comparative clinical studies of PFC-Sigma prostheses design reported no significant difference in evaluation using the 1989 KSS. However, the 1989 KSFS consists of only three items: walking ability, and up or down stairs ability, and use or not of walking aids. There- 
fore, it was felt to not provide enough detail specifically in documenting the functional capabilities of contemporary knee arthroplasty patients. It seems to be more important to consider medium or long-term PRO among different implant design.

Some studies associated with clinical outcomes incorporate perioperative and postoperative research of kinematics using fluoroscopic navigation systems, and of walking analysis using laboratory gait systems. In addition, there is a comparative study of different prosthesis designs in the same patients.

In a previous report that investigated the correlation of clinical outcomes and perioperative kinematics [6], CT-based navigation TKA using PFC Sigma RPF was performed in 40 patients with OA knees. The report showed that the functional activities, patient satisfaction and knee flexion angle in the medial pivot group were significantly better than in the non-medial pivot group, and concluded that an intraoperative medial pivot pattern positively influences deep knee flexion and patient-reported outcomes.

Joglekar et al. [7] reported on 18 subjects with either a PS or CR TKA knees with the PCL sacrificed at surgery and a normal contralateral knee were subjected to physical exam and walking analysis using a staircase model, passive reflective arrays and an opto-electric system. The study showed no significant difference in gait biomechanics during stair ascent or descent when comparing the CR and PS designs.

Kim et al. [8] evaluated clinical outcomes in 66 patients with bilateral TKA using the $\mathrm{MB}$ prosthesis in one knee and the $\mathrm{FB}$ prosthesis in the other at 6,12, and 24 months postoperatively. In addition, the same comparisons between knees fitted with the $\mathrm{MB}$ prosthesis or the $\mathrm{FB}$ prosthesis were made separately in a subgroup of 33 patients implanted with the FB-CR prosthesis and another subgroup of 33 patients with the FB-PS prosthesis. Clinical outcomes in the FB-PS group improved much faster than in the FB-CR group. In contrast, in the FB-CR group, more patients preferred the knee fitted with the MB prosthesis at 6 months. However, in the FB-PS groups, no side preference for the MB prosthesis was observed, and more patients preferred knees implanted with the FB prosthesis at 12 and 24 months. The researchers could not conclude a clear reason for the different intraoperative kinematic patterns against the same patient background.

Furthermore, the effects of TKA on proprioception, kinesthesia, and postural control remain controversial. Some surgeons believe that preserving the PCL may help to preserve sensorimotor function and improve prosthesis longevity and functional outcomes. Swanik et al. [9] evaluated joint-position sense, the threshold to detect joint motion, and standing balance on an unstable platform following TKA with CR and PS TKA preoperatively and at 6 months after surgery for 20 patients. The group treated with the PS prosthesis more accurately reproduced joint position, so retention of the PCL does not appear to significantly improve proprioception and balance. 
In addition, out of four studies reporting patient preference [10] [11] [12] [13], three indicated no significant preference for either bearing [10] [12] [13]. One reported that of 92 bilateral TKA patients, 56 patients (61\%) preferred the $\mathrm{MB}, 6(7 \%)$ the FB, and 30 (33\%) expressed no preference [11].

By contrast, it was reported that several complications occurred with higher frequency in $\mathrm{MB}$ group, including instability and bearing spin-out that required revision [14] [15]. With regard to patello-femoral complications, the incidence of patellar clunk in the PFC Sigma RPF prosthesis has results ranging from $0 \%$ $15 \%$ in the literature [15] [16]. In this study, 3 RP-F knees (3.8\%) and 2 FB-PS knees (2.6\%) had patellar clunk. The decision to use a prosthetic design depends on several factors including the surgeon's preference. Several reviews of the literature have found insufficient evidence to recommend either preservation or substitution of the PCL.

We considered that the restoration of flexion after TKA is crucial for successful clinical outcomes and patient satisfaction, especially in Asian countries where people require the use of high flexion positions. MB high-flexion knee prostheses can be more effective in this regard than FB prostheses due to the design characteristics and rotation of the bearing during hyperflexion [17]. Our analysis showed slightly better ROM using the MB-PS design in comparison to the FB-PS and FB-CR groups. Some articles report no differences between the PS and CR groups regarding ROM, or that postoperative improvement in ROM is significantly superior in the PS group [18] [19]. It is thought that one of the factors associated with flexion limitation in the CR knee is non-physiologic tension of the PCL causing abnormal knee kinematics inflexion. A Cochrane analysis showed a slightly better range of motion using the PS design in comparison to the CR design $\left(113^{\circ}\right.$ vs $\left.105^{\circ}\right)$ [18]. This finding is supported by a number of studies, but the clinical relevancy of a difference in maximal flexion of $8^{\circ}$ is questionable [19]. Moreover, we considered that there are differences between European and Asian populations related to the frequency of the mid to deep flexion positions, including kneeling or squatting in activities of daily living. A functional ROM during daily activity depends on the patient's activity. Therefore, it remains unclear whether a small improvement in the degree of motion will affect patient outcomes; it may instead increase the risk of aseptic loosening or patellar clunk syndrome.

Matsuda et al. [20] reported that varus alignment and limited ROM correlated negatively with patient expectation and functional activities. In our study, there were no significant differences among the three groups related to the 2011 KSKS or radiographic evaluation at last follow-up. In contrast, postoperative 2011 KSFS and expectation scores were significantly higher in the FB- and MB-PS groups than in the FB-CR group. Some influence of the prosthesis design on kinematics during stair-climbing has been reported previously [21]. Therefore, our results seem to indicate an important relationship between prosthesis designs and functional performance. 
There were some potential limitations in the present study. First, this was a retrospective review of our institutional experience using different designs. Second, the final choice of prosthesis may have been modified by the surgeon's preference, thus introducing bias and potentially limiting the value of the comparisons among prosthesis designs in this series. Third, this study did not assess postoperative rotational alignment of the femoral and tibial component by $\mathrm{CT}$ scan. Finally, group numbers were also limited. We believe that we will be able to clarify the difference in performance among different prosthesis designs through further detailed assessment of functional activities.

\section{Conclusion}

This study showed that the PS (MB-PS, FB-PS) prostheses provided significantly better patient-reported functional scores and expectation scores than the CR prostheses. In contrast, postoperative prosthesis-related complication rates were lower with the CR prosthesis. Therefore, we cannot recommend specific prosthesis design over the other at present.

\section{Consent}

We received written informed consent from the patient for publication of the article.

\section{Conflicts of Interest}

The authors declare no conflicts of interest regarding the publication of this paper.

\section{References}

[1] Grupp, T.M., Kaddick, C., Schweisau, J., Maas, A. and Stulberg, S.D. (2009) Fixed and Mobile Bearing Total Knee Arthroplasty-Influence on Wear Generation, Corresponding Wear Areas, Knee Kinematics, and Particle Composition. Clinical Biomechanics, 24, 210-217. https://doi.org/10.1016/j.clinbiomech.2008.11.006

[2] Insall, J.N., Dorr, L.D., Scott, R.D. and Scott, W.N. (1989) Rationale of the Knee Society Clinical Rating System. Clinical Orthopaedics and Related Research, 248, 13-14. https://doi.org/10.1097/00003086-198911000-00004

[3] Scuderi, G.R., Bourne, R.B., Noble, P.C., Benjamin, J.B., Lonner, J.H. and Scott, W.N. (2012) The New Knee Society Knee Scoring System. Clinical Orthopaedics and Related Research, 470, 3-19. https://doi.org/10.1007/s11999-011-2135-0

[4] Roos, E.M., Roos, H.P. and Lohmander, L.S. (1999) WOMAC Osteoarthritis Index-Additional Dimensiouns for Use in Subjects with Post-Traumatic Osteoarthritis of the Knee. Western Ontario and MacMaster Universities. Osteoarthritis Cartilage, 7, 216-221. https://doi.org/10.1053/joca.1998.0153

[5] Ewald, F.C. (1989) The Knee Society Total Knee Arthroplasty Roentgenographic Evaluation and Scoring System. Clinical Orthopaedics and Related Research, 248, 9-12. https://doi.org/10.1097/00003086-198911000-00003

[6] Nishio, Y., Onodera, T., Kasahara, Y., Takahashi, D., Iwasaki, N. and Majima, T. (2014) Intraoperative Medial Pivot Affects Deep Knee Flexion Angle and Pa- 
tient-Reported Outcomes after Total Knee Arthroplasty. The Journal of Arthroplasty, 29, 702-706. https://doi.org/10.1016/j.arth.2013.06.035

[7] Joglekar, S., Gioe, T.J., Yoon, P. and Schwartz, M.H. (2012) Gait Analysis Comparison of Cruciate Retaining and Substituting TKA Following PCL Sacrifice. The Knee, 19, 279-285. https://doi.org/10.1016/j.knee.2011.05.003

[8] Kim, T.K., Chang, C.B., Kang, Y.G., et al. (2010) Early Clinical Outcomes of Floating Platform Mobile-Bearing TKA: Longitudinal Comparison with Fixed-Bearing TKA. Knee Surgery, Sports Traumatology, Arthroscopy, 18, 879-888. https://doi.org/10.1007/s00167-009-0985-8

[9] Swanik, C.B., Lephart, S.M. and Rubash, H.E. (2004) Proprioception, Kinesthesia, and Balance after Total Knee Arthroplasty with Cruciate-Retaining and Posterior Stabilized Prosthesis. The Journal of Bone and Joint Surgery. American Volume, 86, 328-334. https://doi.org/10.2106/00004623-200402000-00016

[10] Price, A.J., Rees, J.L., Beard, D., Juszczak, E., Carter, S., White, S., de Steiger, R., Dodd, C.A., Gibbons, M., Mclardy-Smith, P., Goodfellow, J.W. and Murray, D.W. (2003) A Mobile-Bearing Total Knee Prosthesis Compared with a Fixed-Bearing Prosthesis: A Multicenter Single-Blind Randomized Controlled Trial. The Journal of Bone and Joint Surgery. British Volume, 85, 62-67. https://doi.org/10.1302/0301-620X.85B1.13233

[11] Kim, Y.H., Yoon, S.H. and Kim, J.S. (2009) Early Outcome of TKA with a Medial Pivot Fixed-Bearing Prosthesis Is Worse than with a PFC Mobile-Bearing Prosthesis. Clinical Orthopaedics and Related Research, 467, 493-503. https://doi.org/10.1007/s11999-008-0221-8

[12] Watanabe, T., Tomita, T., Fujii, M., Hashimoto, J., Sugamoto, K. and Yoshikawa, H. (2005) Comparison between Mobile-Bearing and Fixed-Bearing Knees in Bilateral Total Knee Replacements. International Orthopaedics, 29, 179-181. https://doi.org/10.1007/s00264-005-0646-6

[13] Beard, D.J., Pandit, H., Price, A.J., Butler-Manuel, P.A., Dodd, C.A., Murray, D.W. and Goodfellow, J.W. (2007) Introduction of a New Mobile-Bearing Total Knee Prosthesis-Minimum Three Years Follow-Up of an RCT Comparing It with a Fixed-Bearing Device. Knee, 14, 448-451. https://doi.org/10.1016/j.knee.2007.08.004

[14] Aigner, C., Windhager, R., Pechmann, M., Rehak, P. and Engeleke, K. (2004) The Influence of an Anterior-Posterior Glinding Mobile Bearing on Range of Motion after Total Knee Arthroplasty. A Prospective, Randomized, Double-Blinded Study. The Journal of Bone and Joint Surgery. American Volume, 86, 2257-2262. https://doi.org/10.2106/00004623-200410000-00018

[15] Ladermann, A., Lubbeke, A., Stern, R., Riand, N. and Frischy, D. (2008) Fixed-Bearing versus Mobile-Bearing Total Knee Arthroplasty: A Prospective Randomized, Clinical and Radiological Study with Mid-Term Results at 7 Years. Knee, 15, 206-210. https://doi.org/10.1016/j.knee.2008.01.010

[16] Agarwala, S.R., Mohrir, G.S. and Patel, A.G. (2013) Patellar Clunk Syndrome in a Current High Flexion Total Knee Design. The Journal of Arthroplasty, 28, 1846-1850. https://doi.org/10.1016/j.arth.2013.03.019

[17] Nakamura, E., Banks, S.A., Tanaka, A., Sei, A. and Mizuta, H. (2009) Three- Dimensional Tibiofemoral Kinematics during Deep Flexion Kneeling in a Mobile-Bearing Total Knee Arthroplasty. The Journal of Arthroplasty, 24, 1120-1124. https://doi.org/10.1016/j.arth.2008.08.008

[18] Jacobs, W.C., Clement, D.J. and Wymenga, A.B. (2005) Retention versus Sacrifice of the Posterior Cruciate Ligament in Total Knee Replacement for Treatment of Os- 
teoarthritis and Rheumatoid Arthritis. Cochrane Database of Systematic Reviews, 19, CD004803. https://doi.org/10.1002/14651858.CD004803.pub2

[19] Bercik, M.J., Joshi, A. and Parvizi, J. (2013) Posterior Cruciate-Retaining versus Posterior-Stabilized Total Knee Arthroplasty: A Meta-Analysis. The Journal of Arthroplasty, 28, 439-444. https://doi.org/10.1016/j.arth.2012.08.008

[20] Matsuda, S., Kawahara, S., Okazaki, K., Tashio, Y. and Iwamoto, Y. (2013) Postoperative Alignment and ROM Affect Patient Satisfaction after TKA. Clinical Orthopaedics and Related Research, 471, 127-133. https://doi.org/10.1007/s11999-012-2533-y

[21] Banks Scott, A. and Andrew, H.W. (2002) Implant Design Affects Knee Arthroplasty Kinematics during Stair-Stepping. Clinical Orthopaedics and Related Research, 426, 187-193. https://doi.org/10.1097/01.blo.0000138956.04316.ac

\section{Appendix}

The 2011 Knee Society Score: Domains and Point Allocations

Objective Knee Score (seven items, 100 points):

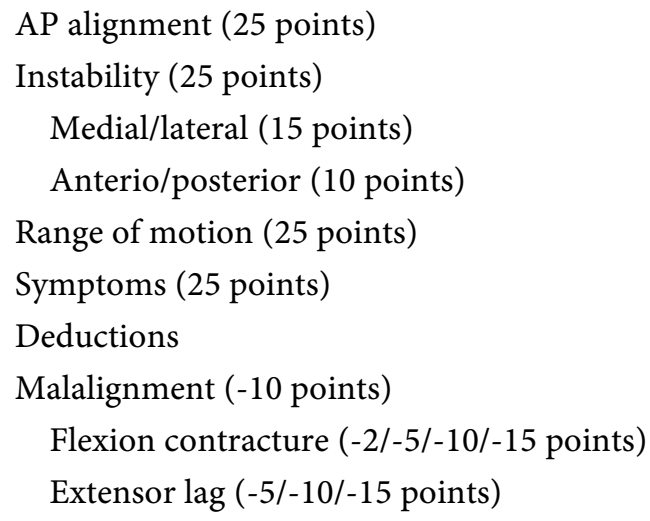

Satisfaction Score (five items, 40 points):

Pain level while sitting (8 points)

Pain level while lying in bed (8 points)

Knee function while getting out of bed (8 points)

Knee function while performing light household duties (8 points)

Knee function while performing leisure recreational activities (8 points)

Expectation score (three items, 15 points):

Pain relief (5 points)

Ability to carry out activities of daily living (5 points)

Ability to perform leisure, recreational, or sports activities (5 points)

Functional Activity Score (19 items, 100 points):

Walking and standing (five items; 30 points)

Standard activities (six items; 30 points)

Advanced activities (five items; 25 points)

Discretionary activities (three items; 15 points) 